\title{
The response of human induced pluripotent stem cells to cyclic temperature changes explored by BIO-AFM
}

\author{
Yan $\mathrm{Nie}^{1,2} \cdot$ Weiwei Wang ${ }^{1} \cdot$ Xun Xu${ }^{1} \cdot \mathrm{Nan}_{\mathrm{Ma}^{1,3}} \cdot$ Andreas Lendlein ${ }^{1,2,3}$
}

Received: 1 April 2021 / Accepted: 9 July 2021 / Published online: 18 August 2021

(c) Helmholtz-Zentrum Hereon 2021

\begin{abstract}
Human induced pluripotent stem cells (hiPSCs) are highly sensitive to extrinsic physical and biochemical signals from their extracellular microenvironments. In this study, we analyzed the effect of cyclic temperature changes on hiPSCs behaviors, especially by means of scanning force microscopy (BIO-AFM). The alternation in cellular mechanics, as well as the secretion and pattern of deposition of extracellular matrix (ECM) protein in hiPSCs were evaluated. The arrangement of the actin cytoskeleton changed with the variation of the temperature. The rearranged cytoskeleton architecture led to the subsequent changes in cell mechanics (Young's modulus of hiPSCs). With the exposure to the cyclic cold stimuli, an increase in the average surface roughness (Ra) and roughness mean square (RMS) was detected. This observation might be at least in part due to the upregulated secretion of Laminin $\alpha 5$ during repeated temporary cooling. The expression of pluripotent markers, NANOG and SOX2, was not impaired in hiPSCs, when exposed to the cyclic cold stimuli for $24 \mathrm{~h}$. Our findings provide an insight into the effect of temperature on the hiPSC behaviors, which may contribute to a better understanding of the application of locally controlled therapeutic hypothermia.
\end{abstract}

\section{Introduction}

Human induced pluripotent stem cells (hiPSCs)-based models are promising tools for the investigation of stem cell fate and the development of patient-specific diseases models [1, 2]. In their extracellular environment, hiPSCs are exposed to multiple mechanical stimuli that regulate their fate [3-5]. As pluripotent cells, hiPSCs are able to adapt to those stresses and prevent themselves from damage [6,7]. It showed that

Nan Ma was an editor of this journal during the review and decision stage. For the MRS Advances policy on review and publication of manuscripts authored by editors, please refer to mrs. org/editor-manuscripts.

Nan Ma

nan.ma@hereon.de

Andreas Lendlein

andreas.lendlein@hereon.de

1 Institute of Active Polymers and Berlin-Brandenburg Centre for Regenerative Therapies, Helmholtz-Zentrum Hereon, 14513 Teltow, Germany

2 Institute for Biochemistry and Biology, University of Potsdam, Potsdam, Germany

3 Institute of Chemistry and Biochemistry, Free University of Berlin, Berlin, Germany mechanosensitive ion channels were able to detect and channel the stresses [8], as the activated channels allowed the influx of cations and led to the cytoskeleton remodeling [9, 10]. The downstream signaling crosstalk, such as the Hippo/ YAP pathway and Ras-activated signaling pathway, was then activated to counteract the stress [11-13].

Another important stress response of the cells is the thermal response $[14,15]$. Cells have a variety of adaptive mechanisms to withstand temperature changes [16]. Heat stress is encountered in numerous medical conditions, such as inflammation [17], which led to the activation of heat shock proteins $[18,19]$. Cold stress resulted in losing the cell membrane permeability, altering the enzyme activity, and modifying the cytoskeletons [20-23]. It should be noted that the temperature affects the supramolecular structures and conformations of extracellular matrix (ECM) proteins [24]. Temperature change (from 98 to $320 \mathrm{~K}$ ) led to an expansion of total volume of protein $(0.4 \%$ per $100 \mathrm{~K})$ [25]. In the solution, the assembly of ECM protein was favored by increased temperature, as the attraction to the net force increases with temperature from 5 to $35^{\circ} \mathrm{C}$ [26]. Consequently, cellular behaviors were altered by changes in ECM [27]. It is intrigued that the cold responses was not the same among different animal species, and the molecular basis in cold adaption is remained to be elucidated [23, 28]. Here, we 
examined the influence of cyclic temperature changes $(\Delta \mathrm{T})$ on the interaction between the cells and the ECM, including the cell shape and mechanical properties as well as the ECM topography.

\section{Method}

Detailed information regarding the BIH-001A cell line, cell culture, and fluorescence staining appear in Supplementary Method S1. The topography of decellularized extracellular matrix (dECM) and the mechanics of the hiPSCs were determined using an atomic force microscope (NanoWizard 4, JPK BioAFM, Germany). Decellularization was using the following protocol [29]. Briefly, samples were washed with $0.1 \%$ (w/v) sodium dodecyl sulfate (Sigma-Aldrich, Germany) for $1 \mathrm{~h}$ at room temperature. DNA contents were removed using $10 \mathrm{U} / \mathrm{ml}$ DNAse (Sigma-Aldrich, Germany). The topography of the AECM was measured using AC Mode in PBS at room temperature. The experiments involving living cells were conducted at $37 \pm 0.1{ }^{\circ} \mathrm{C}$ controlled by a petri dish heater (JPK BioAFM, Germany). A tetrahedral tip (240-AC-NG, NanoAndMore GmbH, Germany) was used to measure the topography of the $\mathrm{dECM}$ and a colloidal tip (CP-qp-CONT-Au-B-5, NanoAndMore GmBH, Germany) to measure Young's modulus of a single living hiPSC (Fig. $\mathrm{S} 1$ ). Images and force curves were recorded by the JPKSPM software (JPK BioAFM, Germany) and were processed using JPKSPM Data Processing software (JPK BioAFM, Germany). Detailed descriptions appear in the supplemental information.

\section{Statistical analysis}

Data were expressed as mean \pm standard deviation (SD). GraphPad Prism 8 (GraphPad Software, USA) was used for the statistical analysis using unpaired $t$-tests. $p<0.05$ was considered statistically significant.

\section{Results and discussion}

\section{Pluripotency of hiPSCs in response to cyclic temperature changes}

The expression of NANOG and SOX2 was used to evaluate the pluripotency of hiPSCs (Fig. 1). A robust expression of the NANOG and SOX2 was observed, while an increase in SOX2 expression was detected in hiPSCs under the cyclic cold stress. As either higher or lower expression of SOX2 would lead to the loss of pluripotency [30, 31], our result implied that the cyclic cold stress might favor the ectodermal lineage commitment in hiPSCs through upregulation SOX2 [32-34]. In addition, the cell-intrinsic mechanics regulated the pluripotent state of the cells [35], this observation might also due to the changes in mechanical properties of hiPSCs.

\section{Alterations in the rearrangement of cytoskeleton caused by cyclic temperature changes}

The ability of a eukaryotic cell to maintain or change its shape depends on the cytoskeleton arrangement
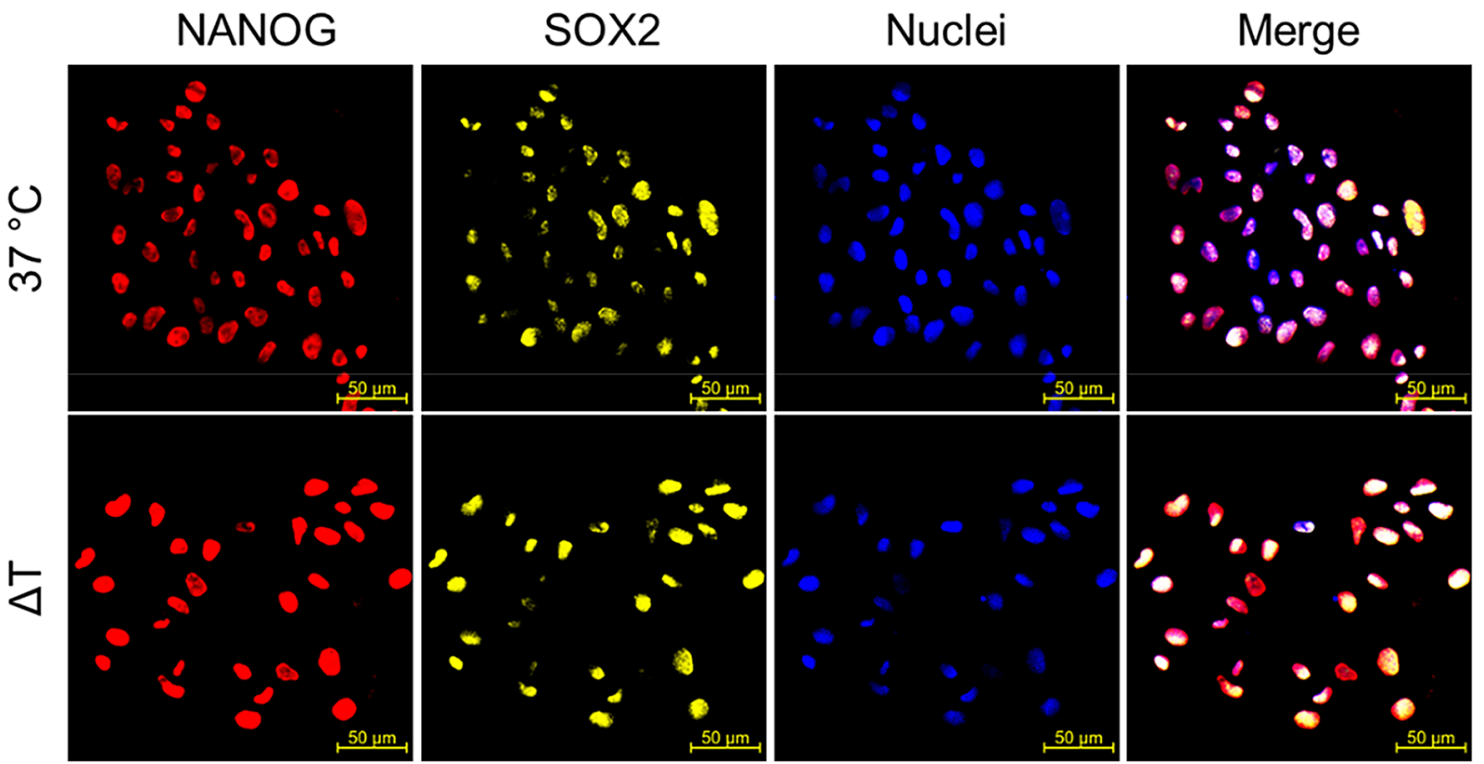

Fig. 1 Representative images of pluripotent markers expressed in hiPSCs with or without exposure to cyclic temperature changes for $24 \mathrm{~h}$ (scale bars are $50 \mu \mathrm{m})$ 
[36]. Actin filament (F-actin) is one of the main types of cytoskeletal polymers, which regulates cell shape, mechanical properties, and intracellular signaling transduction [37]. Our result showed that, in both groups, thick F-actin fibers were displayed in parallel to the colony edge of hiPSCs, while a stronger fluorescence signal was detected in the hiPSC colony exposed to the cyclic temperature changes (Fig. 2a). At the single-cell level, more filopodia that formed by the long tight bundles of F-actin were observed in hiPSCs treated with the repeated temporary cooling. Without temperature change, the lamellipodia and cortical actin meshwork became apparent.

\section{Alterations in the mechanics of hiPSCs treated with the cold stimuli}

The cellular mechanical properties are dependent on the integrity and organization of the cytoskeleton [38]. We have demonstrated that the temperature change was a potent regulator of the expression and architecture of cytoskeleton component in the adult cells [39]. The value of Young's modulus in hiPSCs subjected to the cyclic temperature change was increased (Fig. 2b). The distribution of Young's modulus in hiPSCs at $37^{\circ} \mathrm{C}$ was narrower than those exposed to the cyclic cold stimuli. The temperature-induced stiffening of hiPSCs was a result of the altered cytoskeleton arrangement [40, 41].

\section{ECM remodeled by hiPSCs in response to cold stress}

Through regulating the synthesis and secretion, assembly and reassembly, chemical modification, and degradation, ECM is constantly modified by the cells [42]. As the ECM become more and more stable along with its maturation, the remodeling of matured ECM would require proteases [43]. In addition, temperature change could affect the ECM assembly in the absence of cells [26]. The ECM topography was measured to reflect thermal influence on the capacity of hiPSCs to remodel their ECM using AFM (Fig. 3a). More surface incongruity was detected in the $\mathrm{dECM}$ derived from the cyclic cold-stimulated hiPSCs, as irregular larger peaks (the bright regions) with approximately $2 \mu \mathrm{m}$ in width and $40 \mathrm{~nm}$ in height were observed.

The roughness average ( $\mathrm{Ra})$ and the root mean square (RMS) values were chosen to quantitatively characterize the surface roughness of the dECM (Table 1). In dECM derived from the hiPSCs exposed to the repeated temporary cooling, a significantly higher degree of roughness was exhibited. Previous studies demonstrated that cells could generate tractional forces through the cytoskeleton deformation to regulate the architecture of ECM. As the repeated temporary cooling altered the hiPSC cytoskeleton [44], it might thus cause changes in ECM arrangement.

Another possible reason for the altered ECM architecture is the production of ECM proteins by the cells. Laminin is one of the main components of ECM that involved in cellECM interactions and ECM remodeling [45, 46]. A substantial increase of Laminin $\alpha 5$ secretion was detected in hiPSCs
Fig. 2 Effects of cyclic temperature changes on hiPSC morphology and mechanical properties. a Representative images of F-actin organization in the hiPSC colony or in a single hiPSC cultured under different conditions for $24 \mathrm{~h}$ (scale bars for the image of hiPSC colony are $100 \mu \mathrm{m}$, for the single hiPSC are $50 \mu \mathrm{m})$. b Young's modulus of hiPSCs. Fifty-five single cells (biological replicates) were measured, each cell was measured three times (technical replicates), together 165 force curves were acquired for calculating Young's modulus of hiPSCs $(n=165$; $* p<0.0001)$

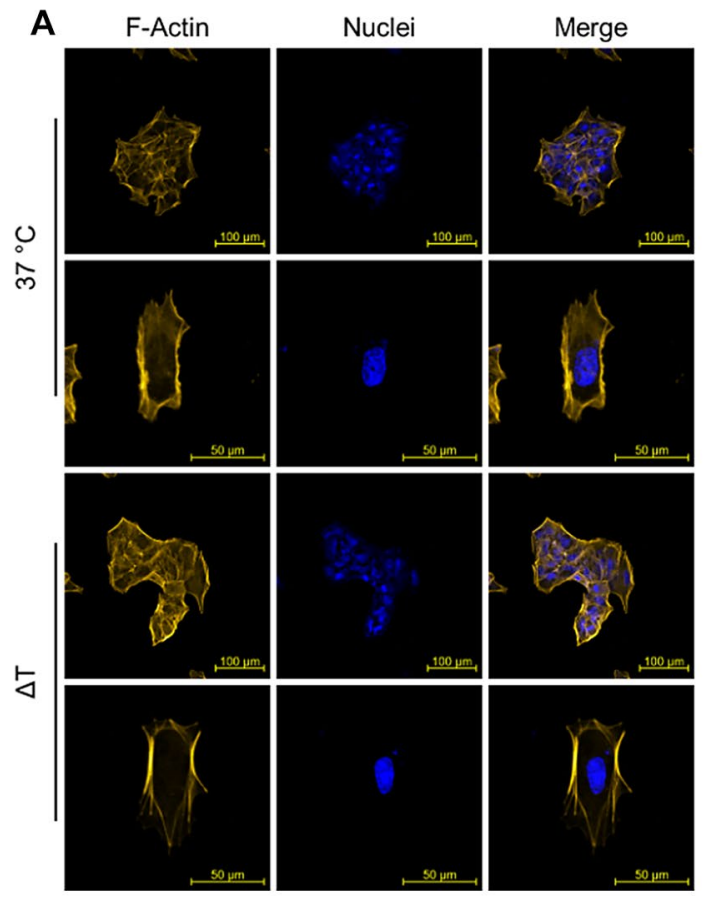

B

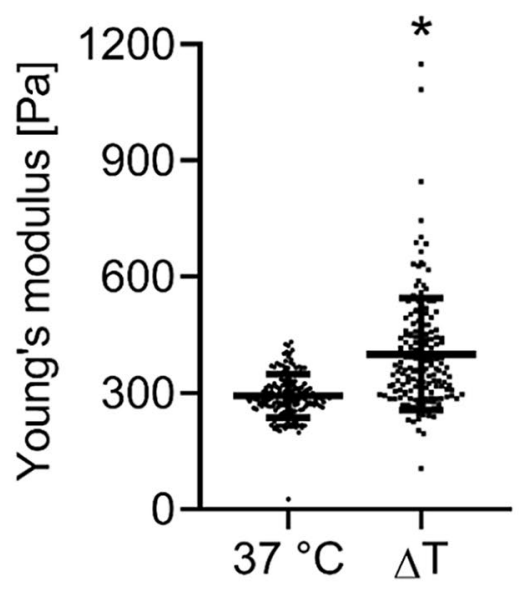


A

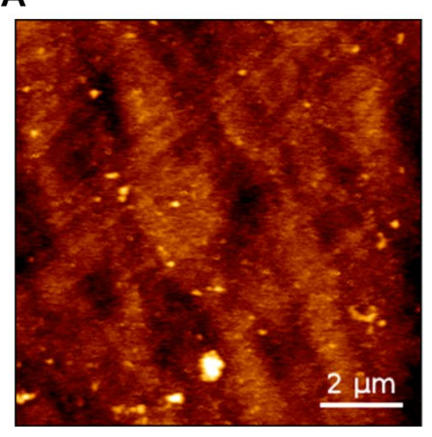

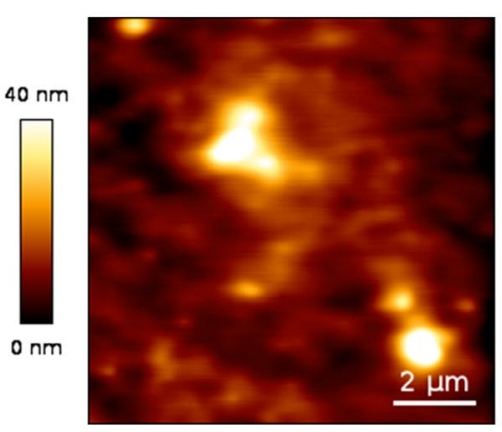

B

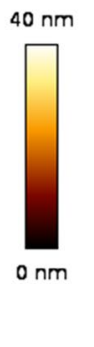

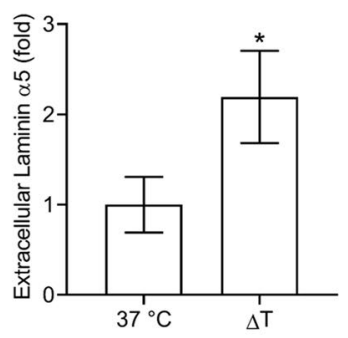

Fig. 3 ECM remodeled by hiPSCs in response to cyclic temperature changes. a Representative topography of dECM derived from hiPSCs with or without the cyclic temperature changes for $24 \mathrm{~h}$. b Fold

Table 1 Mean \pm standard deviation of Ra and RMS values for the dECM samples $(n=10, * p<0.0005)$

\begin{tabular}{lll}
\hline Culture condition & $\mathrm{Ra}(\mathrm{nm})$ & $\mathrm{RMS}(\mathrm{nm})$ \\
\hline $37^{\circ} \mathrm{C}$ & $31.56 \pm 2.82$ & $38.07 \pm 3.58^{*}$ \\
$\Delta \mathrm{T}$ & $39.52 \pm 2.83$ & $49.14 \pm 4.23^{*}$ \\
\hline
\end{tabular}

exposed to the cyclic cold stimuli (Fig. 3b). The difference in the secretion mode of Laminin $\alpha 5$ might be a result of the altered the arrangement of the cytoskeleton, as the actin cytoskeleton could play a regulatory role in the ECM protein secretion [47].

\section{Conclusion}

In this study, we assessed the responses of hiPSCs to the cyclic temperature changes, including the pluripotency, the cytoskeleton arrangement, and Young's modulus of the hiPSCs as well as their ability to remodel the ECM. Cyclic temperature changes did not decrease the expression of pluripotent proteins in hiPSCs, while it caused alterations in the arrangement of the actin skeleton, which further altered the ability of hiPSCs to remodel the ECM. The AECM in the group with cyclic temperature change presented increased surface roughness. These results provide insight into how hiPSCs adapt to the signals in the ambient environment, which might deepen the understanding of the cold-adaptive behaviors in stem cells and uncover the potential of therapeutic hypothermia.

Supplementary Information The online version contains supplementary material available at https://doi.org/10.1557/s43580-021-00110-4.

Acknowledgments We thank the Berlin Institute of Health (BIH) Stem Cell Core Facility and Dr. Stachelscheid for kindly providing us with hiPSC line BIHi001-A. This work was financially supported by the changes in the secretion of Laminin $\alpha 5$ in hiPSCs exposed to cyclic temperature changes for $24 \mathrm{~h}(n=3 ; * p<0.05)$

Helmholtz Association of German Research Centers through programoriented funding as well as the Federal Ministry of Education and Research, Germany, for funding through the Program Health Research (Grant No. 13GW0098). Yan Nie is grateful for the support from the China Scholarship Council (CSC, No. 201706205049).

Funding Open Access funding enabled and organized by Projekt DEAL.

Availability of data Data will be made available on reasonable request.

\section{Declarations}

Conflict of interest The authors declare no competing financial interest.

Open Access This article is licensed under a Creative Commons Attribution 4.0 International License, which permits use, sharing, adaptation, distribution and reproduction in any medium or format, as long as you give appropriate credit to the original author(s) and the source, provide a link to the Creative Commons licence, and indicate if changes were made. The images or other third party material in this article are included in the article's Creative Commons licence, unless indicated otherwise in a credit line to the material. If material is not included in the article's Creative Commons licence and your intended use is not permitted by statutory regulation or exceeds the permitted use, you will need to obtain permission directly from the copyright holder. To view a copy of this licence, visit http://creativecommons.org/licenses/by/4.0/.

\section{References}

1. A. Mitchell, H. Wanczyk, T. Jensen, C. Finck, Am. J. Transl. Res. 12(1), 292 (2020)

2. C. Fan, E. Zhang, J. Joshi, J. Yang, J. Zhang, W. Zhu, Front. Cell Dev. Biol. 8, 36 (2020)

3. R. Goetzke, A. Sechi, L. De Laporte, S. Neuss, W. Wagner, Cell. Mol. Life Sci. 75(18), 3297 (2018)

4. F. Zhang, J. Wang, D. Lü, L. Zheng, B. Shangguan, Y. Gao, Y. Wu and M. Long, Biomechanics and Modeling in Mechanobiology, 1 (2020). 
5. I. Horiguchi, F.G. Torizal, H. Nagate, H. Inose, K. Inamura, O. Hirata, H. Hayashi, M. Horikawa, Y. Sakai, Biotechnol. Progr. 37, e3100 (2020)

6. R. Ohtani-Kaneko, K. Sato, A. Tsutiya, Y. Nakagawa, K. Hashizume, H. Tazawa, Biomed. Microdevice 19(4), 1 (2017)

7. T.J. Kolanowski, M. Busek, M. Schubert, A. Dmitrieva, B. Binnewerg, J. Pöche, K. Fisher, F. Schmieder, S. Grünzner, S. Hansen, Acta Biomater. 102, 273 (2020)

8. P. Jin, L.Y. Jan, Y.-N. Jan, Annu. Rev. Neurosci. 43, 207 (2020)

9. C. Zhang, M. Skamagki, Z. Liu, A. Ananthanarayanan, R. Zhao, H. Li, K. Kim, Cell Rep. 21(8), 2058 (2017)

10. L. He, M. Ahmad, N. Perrimon, Exp. Cell Res. 374(2), 259 (2019)

11. B. Oh, Y.W. Wu, V. Swaminathan, V. Lam, J. Ding, P.M. George, Adv. Sci. 8, 2002112 (2021)

12. J. Yang, C. Wu, I. Stefanescu, L. Jakobsson, I. Chervoneva, A. Horowitz, Sci. Signal. 9(438), 76 (2016)

13. L. Macri-Pellizzeri, B. Pelacho, A. Sancho, O. Iglesias-Garcia, A.M. Simón-Yarza, M. Soriano-Navarro, S. González-Granero, J.M. García-Verdugo, E.M. De-Juan-Pardo, F. Prosper, Tissue Eng. Part A 21(9-10), 1633 (2015)

14. C.J. Hunt, Transf. Med. Hemotherapy 46(3), 134 (2019)

15. J. Zhang, C. Cheng, J.L. Cuellar-Camacho, M. Li, Y. Xia, W. Li, R. Haag, Adv. Funct. Mater. 28(47), 1804773 (2018)

16. H.N. Pawar, G.R. Kumar, R. Narang, R.K. Agrawal, Int. J. Curr. Microbiol. App. Sci. 3(2), 307 (2014)

17. P. Shende, S. Bhandarkar, B. Prabhakar, Stem Cell Rev. Rep. 15(5), 637 (2019)

18. M.K. Singh, B. Sharma, P.K. Tiwari, J. Therm. Biol 69, 149 (2017)

19. A.D. Zuehlke, M.A. Moses, L. Neckers, Philos. Trans. R. Soc. B 373(1738), 20160527 (2018)

20. E. Saita, D. Albanesi, D. de Mendoza, Biochimica et Biophysica Acta (BBA) 1861(8), 837 (2016)

21. Y. Abe, Y. Fujiwara, H. Takahashi, Y. Matsumura, T. Sawada, S. Jiang, R. Nakaki, A. Uchida, N. Nagao, M. Naito, Nat. Commun. 9(1), 1 (2018)

22. Q. Zhang, Y.-Z. Wang, W. Zhang, X. Chen, J. Wang, J. Chen, W. Luo, Mol. Neurobiol. 54(3), 2143 (2017)

23. A. Roobol, M.J. Carden, R.J. Newsam, C.M. Smales, FEBS J. 276(1), 286 (2009)

24. Y.-C. Yu, P. Berndt, M. Tirrell, G.B. Fields, J. Am. Chem. Soc. 118(50), 12515 (1996)

25. R.F. Tilton Jr., J.C. Dewan, G.A. Petsko, Biochemistry 31(9), 2469 (1992)
26. S. Leikin, D. Rau, V. Parsegian, Proc. Natl. Acad. Sci. 91(1), 276 (1994)

27. B.J. Dzamba, D.W. DeSimone, Curr. Top. Dev. Biol. 130, 245 (2018)

28. J. Chen, D. Kang, J. Xu, M. Lake, J.O. Hogan, C. Sun, K. Walter, B. Yao, D. Kim, Nat. Commun. 4(1), 1 (2013)

29. I. Ullah, R. Abu-Dawud, J.F. Busch, A. Rabien, B. Erguen, I. Fischer, P. Reinke, A. Kurtz, Biomaterials 216, 119283 (2019)

30. S. Zhang, W. Cui, World J. Stem Cells 6(3), 305 (2014)

31. Z. Wang, E. Oron, B. Nelson, S. Razis, N. Ivanova, Cell Stem Cell 10(4), 440 (2012)

32. M. Behbahaninia, W.L. Ramey, M.K. Sindhwani, M.Y.S. Kalani, Neurosurgery 69(4), N19 (2011)

33. G. Driessens, C. Blanpain, Cell Stem Cell 9(4), 283 (2011)

34. J.L. Kopp, B.D. Ormsbee, M. Desler, A. Rizzino, Stem Cells 26(4), 903 (2008)

35. M. Bergert, S. Lembo, S. Sharma, L. Russo, D. Milovanović, K.H. Gretarsson, M. Börmel, P.A. Neveu, J.A. Hackett, E. Petsalaki, Cell Stem Cell 28(2), 209 (2021)

36. C. Luxenburg, R. Zaidel-Bar, Exp. Cell Res. 378(2), 232 (2019)

37. P.P. Provenzano, P.J. Keely, J. Cell Sci. 124(8), 1195 (2011)

38. A.F. Pegoraro, P. Janmey, D.A. Weitz, Cold Spring Harbor Perspect. Biol. 9(11), 22038 (2017)

39. Y. Nie, X. Xu, W. Wang, N. Ma, A. Lendlein, Clin. Hemorheol. Microcirc. 1, 1 (2020)

40. A. Pocaterra, G. Santinon, P. Romani, I. Brian, A. Dimitracopoulos, A. Ghisleni, A. Carnicer-Lombarte, M. Forcato, P. Braghetta, M. Montagner, J. Hepatol. 71(1), 130 (2019)

41. M.L. Gardel, F. Nakamura, J.H. Hartwig, J.C. Crocker, T.P. Stossel, D.A. Weitz, Proc. Natl. Acad. Sci. 103(6), 1762 (2006)

42. J. Chang, M.-H. Kim, E. Agung, S. Senda, M. Kino-Oka, Regenerat. Therapy 10, 27 (2019)

43. S.L. Dallas, Q. Chen, P. Sivakumar, Curr. Top. Dev. Biol. 75, 1 (2006)

44. B.A. Krajina, B.L. LeSavage, J.G. Roth, A.W. Zhu, P.C. Cai, A.J. Spakowitz, S.C. Heilshorn, Sci. Adv. 7(8), 1969 (2021)

45. T.H. Perera, X. Lu, L.A. Smith Callahan, J. Funct. Biomater. 11(1), 15 (2020)

46. A. Hyysalo, M. Ristola, M.E.-L. Mäkinen, S. Häyrynen, M. Nykter, S. Narkilahti, Stem Cell Res. 24, 118 (2017)

47. L.H. Jørgensen, P.L. Jepsen, A. Boysen, L.B. Dalgaard, L.G. Hvid, N. Ørtenblad, D. Ravn, J. Sellathurai, J. Møller-Jensen, H. Lochmüller, Am. J. Pathol. 187(2), 457 (2017) 